\title{
Task Switching and Novelty Processing Activate a Common Neural Network for Cognitive Control
}

\author{
Francisco Barcelo ${ }^{1}$, Carles Escera ${ }^{2}$, Maria J. Corral ${ }^{2}$, \\ and Jose A. Periáñez ${ }^{1}$
}

\begin{abstract}
The abrupt onset of a novel event captures attention away from, and disrupts, ongoing task performance. Less obvious is that intentional task switching compares with novelty-induced behavioral distraction. Here we explore the hypothesis that intentional task switching and attentional capture by a novel distracter both activate a common neural network involved in processing contextual novelty [Barcelo, F., Periáñez, J. A., \& Knight, R. T. Think differently: A brain orienting response to task novelty. NeuroReport, 13, 1887-1892, 2002.]. Eventrelated potentials were recorded in two task-cueing paradigms while 16 subjects sorted cards following either two (color or shape; two-task condition) or three (color, shape, or number; three-task condition) rules of action. Each card was preceded by a familiar tone cueing the subject either to switch or to repeat the previous rule. Novel sound distracters were interspersed in one of two blocks of trials in each condition. Both novel sounds and task-switch cues impaired responses to the following visual target. Novel sounds elicited novelty P3
\end{abstract}

potentials with their usual peak latency and frontal-central scalp distribution. Familiar tonal switch cues in the three- and two-task conditions elicited brain potentials with a similar latency and morphology as the novelty $\mathrm{P} 3$, but with relatively smaller amplitudes over frontal scalp regions. Covariance and principal component analyses revealed a sustained frontal negative potential that was distorting concurrent novelty $\mathrm{P} 3$ activity to the tonal switch cues. When this frontal negativity was statistically removed, P3 potentials to novel sounds and taskswitch cues showed similar scalp topographies. The degree of activation in the novelty $\mathrm{P} 3$ network seemed to be a function of the information (entropy) conveyed by the eliciting stimulus for response selection, over and above its relative novelty, probability of occurrence, task relevance, or feedback value. We conclude that novelty $\mathrm{P} 3$ reflects transient activation in a neural network involved in updating task set information for goal-directed action selection and might thus constitute one key element in a central bottleneck for attentional control.

\section{INTRODUCTION}

A major goal of modern neuroscience is to understand the precise neural mechanisms that underlie the control of human cognition (Ridderinkhof, van den Wildenberg, Segalowitz, \& Carter, 2004; Rushworth, Walton, Kennerley, \& Bannerman, 2004; Braver, Reynolds, \& Donaldson, 2003; Koechlin, Ody, \& Kouneiher, 2003; Corbetta \& Shulman, 2002; Shallice, 2002; Duncan \& Owen, 2000; Carter et al., 1998). Cognitive control can be accomplished externally by salient sensory events (stimulus-driven control); for instance, when an alarm bell makes you stop and turn towards a potential hazard. Cognitive control can also be enforced internally by current plans and goals of action (goal-directed control); for instance, when you recall the steps to cook your favorite meal. Although inextricably intertwined, the exogenous and endogenous controls of cognition have normally been examined using rather distinct task procedures. Behav-

${ }^{1}$ University of Illes Balears, Palma de Mallorca, Spain, ${ }^{2}$ University of Barcelona, Spain

ioral distraction paradigms have been used to explore exogenous control, where abrupt onsets of contextually deviant or novel events divert attention away and impair performance in a main task (de Fockert, Rees, Frith, \& Lavie, 2004; Ranganath \& Rainier, 2003; Escera, Alho, Winkler, \& Näätänen, 1998). The endogenous control of cognition has been appraised using task-switching paradigms that demand intentional shifts in attention linked to the occurrence of some prelearned set of contextual cues (Brass \& von Cramon, 2004; Monsell, 2003; Rushworth, Passingham, \& Nobre, 2002). Task switching involves the selection, rehearsal, and updating of task-set information in working memory, and it has been regarded as a good benchmark for exploring the neural mechanisms of the goal-directed control of human attention (Corbetta \& Shulman, 2002; Rushworth et al., 2002).

Models of human cognition often portray behavioral distraction and task switching as two distinct forms of cognitive control, examined with different task procedures, and accounted for from separate theoretical frameworks (Pashler, Johnston, \& Ruthruff, 2001; Yantis, 2000). However, indirect evidence suggests that goal-directed 
and intentional control of task switching shares a common neural substrate with the appraisal of rare or novel distracters (Barcelo, Periáñez, \& Knight, 2002). Contextually novel events that capture attention away from an ongoing task elicit an electrical brain potential often referred to as the "novelty P3" (Figure 1C and D) (cf., Ranganath \& Rainier, 2003; Friedman, Cycowicz, \& Gaeta, 2001). The novelty P3 is often taken to indicate that a novel distracter has captured attention and, at that point in time, is most likely within the focus of mind (Ranganath \& Rainier, 2003). Recent indirect evidence suggests that familiar and repetitive contextual cues that announce a switch to a new task activate the same network of brain regions implicated in processing taskirrelevant novel distracters (Barcelo et al., 2002). In the study by Barcelo et al. (2002), the morphology, latency, and frontal-central distribution of $\mathrm{P} 3$ responses to familiar task-switch cues (hereafter the "task-novelty P3") were reminiscent of the morphology, latency, and frontalcentral distribution of $\mathrm{P} 3$ responses to novel distracters (hereafter the "stimulus-novelty P3") ${ }^{1}$ (Ranganath \& Rainier, 2003; Escera et al., 1998). Accordingly, the authors concluded that the appraisal of a new task recruits activity in the same network of brain regions as the appraisal of a novel distracter, an idea referred to as the "task novelty hypothesis" (Barcelo et al., 2002). Such a proposal is in accord with several other pieces of indirect evidence. First, exogenous and endogenous modes of attentional control appear to be subserved by partially overlapping neural networks (de Fockert et al., 2004; Nobre, 2001; Duncan \& Owen, 2000). Second, a substantial body of evidence suggests a constant interaction between both forms of cognitive control (Pashler et al., 2001; Yantis, 2000). Even if the abrupt onset of a new object may capture attention in an automatic, stimulusdriven fashion (Ranganath \& Rainier, 2003; Escera et al., 1998), this obligatory mechanism may be subject to modulation from top-down attentional control (Escera, Yago, Corral, Corbera, \& Nunez, 2003; Sussman, Winkler, \& Schroger, 2003; Yantis, 2000). Such interactions could be best explained by a partial overlap in the neural networks subserving stimulus-driven and goal-directed modes of operation. Finally, it has been well established that motor responses are slower and more error-prone after a switch in task (Monsell, 2003; Rubinstein, Meyer, \& Evans, 2001), a phenomenon reminiscent of the disruptions in attentional efficiency caused by novel distracters. Despite these hints, so far no study has directly addressed whether task switching and behavioral distraction by novel events both activate a common network of brain regions (cf., Posner, 2004).

The nature of the interactions between goal-directed and stimulus-driven control of cognition, as well as their putative neural substrates, has remained elusive partly
Figure 1. Task design, and main behavioral and ERP results. (A) Schematic example of one trial in our task-cueing protocol. Tonal "switch" cues signaled a change in task, whereas "repeat" cues prompted the subject to repeat the previous task. The pitch of switch and repeat cues was counterbalanced across subjects $(500$ and $1000 \mathrm{~Hz}$, or vice versa). The target card remained on display until a response was given. Novel sound distracters were randomly interspersed in one block of trials $(200 \mathrm{msec}$ duration; $10 \mathrm{msec}$ rise/fall times; $75 \mathrm{~dB}$ SPL for all auditory stimuli). (B) Mean RTs to target cards following a task-switch cue, a task-repeat cue, and a novel sound distracter in both the two-task and three-task conditions. (C) Grand-average ERPs recorded at three midline electrodes in response to task-switch and task-repeat cues in the three-task (purple line) and two-task (green line) conditions. The grand ERP average to the novel sounds (red line) is compared with the task-switch cues. (D) Spline-interpolated scalp maps of brain responses to novel sounds and switch cues in the three-task and two-task conditions, both for mean P3 voltages (upper row) and N1-corrected P3 voltages (lower row).

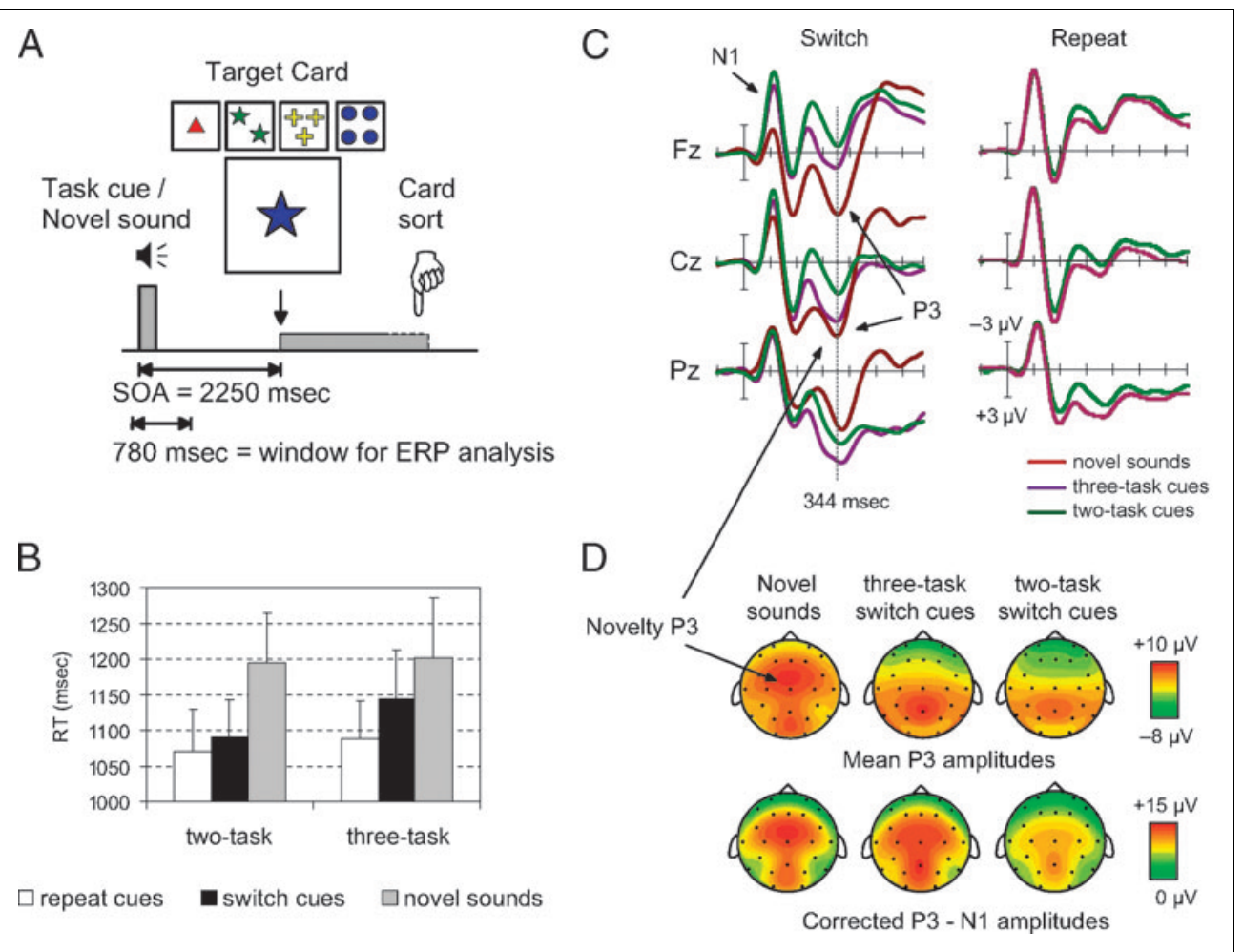

Barcelo et al. 
due to methodological difficulties in extricating the influence of the current task set (Pashler et al., 2001; Yantis, 2000). The vast majority of studies on the neural bases of goal-directed control has searched for brain activations in response to task-relevant and repetitive target events in settings that favor task-set maintenance and the build-up of a prepotent stimulus-response mapping (Posner, 2004; Corbetta \& Shulman, 2002). In contrast, the neural bases of stimulus-driven control have been explored using rare or unique task-irrelevant distracters that generate attentional switches away from a main task (de Fockert et al., 2004; Escera, Alho, Schröger, \& Winkler, 2000; Escera et al., 1998). In both cases, the experimental settings typically consist of single task-set paradigms (like "oddball" tasks, i.e., "count the red bars"), where goal-directed control is normally equated with task-set maintenance operations. In such conditions, the brain responses elicited by task-irrelevant distracters are typically interpreted as stimulus-driven or exogenously generated (Polich, 2003; Friedman et al., 2001). In our view, this is a biased interpretation because single task-set paradigms are not designed to discern between brain activations caused by stimulus-driven or goal-directed task-set switching operations. A more adequate control for the brain activations caused by onsets of a task-irrelevant novel distracter requires the use of task-relevant familiar cues that signal either an intentional shift in set ("task-switch" cues), or the maintenance of the same set ("taskrepeat" cues). ${ }^{2}$ To our knowledge, there have been no prior attempts to compare the brain responses to taskirrelevant novel distracters with those evoked by familiar contextual cues signaling either goal-directed switches or repetitions in the current task set (cf., Posner, 2004). In doing so, here we examined the "task novelty" hypothesis that processing of novel tasks and novel distracters activates a common network of brain regions involved in cognitive control (Barcelo et al., 2002).

To test this hypothesis, we used scalp-recorded brain potentials to examine, at a network level, the fast dynamics of neural responses time-locked to novel sound distracters and familiar task-relevant tonal cues in a well-known task-set switching paradigm (Figure 1A) (Rubinstein et al., 2001; Milner, 1963). The task novelty hypothesis was formulated on cue-locked P3-like activity, as functionally distinct from target-locked $\mathrm{P} 3 \mathrm{~b}$ responses (Barcelo, 2003; Barcelo et al., 2002). The subjects' task was to classify a choice card following either two (color or shape; "two-task condition") or three (color, shape, or number; "three-task condition") rules of action. Prior to target onset, a prelearned familiar tonal cue informed whether to switch or to repeat the previous rule. Tonal switch and repeat cues occurred unpredictably in the two-task (switch probability $=.5$ ) and three-task conditions (switch probability $=.4$ ) (cf., Barcelo, et al., 2002). Novel and highly unfamiliar sound distracters were randomly interspersed in one of two blocks of trials in each task condition (Escera et al., 2003). These two conditions were intended to replicate our previous results (Barcelo et al., 2002) and to ascertain alternative accounts of task-novelty $\mathrm{P} 3$ activity in terms of an orienting response to the infrequent "oddball" cues or to the negative feedback signals (Dien, Spencer, $\&$ Donchin, 2004). We made two predictions in line with the points raised previously. First, if goal-directed taskset switching compares with stimulus-driven attentional capture by novel distracters, then familiar tonal cues that signal a change to a new task will elicit brain responses with similar morphology, latency, and scalp distribution as the stimulus-novelty P3. This first prediction was assessed by examining the scalp distribution of P3 potentials to the auditory events. The absence of an interaction between auditory event (i.e., two-task cues, three-task cues, novel sounds) and electrode site of recording would suggest a similar scalp distribution of the P3 potentials to these auditory events, hence, a common distributed network of neural generators (McCarthy \& Wood, 1985; Picton, Woods, Stuss, \& Campbell, 1978). Second, we also expected that taskrelevant tonal cues and task-irrelevant novel sounds would elicit partly different brain responses. This prediction was made on purely theoretical grounds. We reasoned that task-set information would exert a top-down influence on the brain responses to within-set tonal cues, whereas such modulations would be absent from the brain responses to out-of-set novel distracters. In line with existing models, we predicted such top-down influences mainly upon the endogenous, late eventrelated potential (ERP) components to the tonal cues (Hillyard, Mangun, Woldorff, \& Luck, 1995; Näätänen, 1990).

\section{METHODS}

\section{Subjects}

Sixteen young subjects (mean age $21 \pm 0.6$ years, range 19-26 years; 10 women) took part in the study. They all had normal or corrected-to-normal vision and no history of neurological or psychiatric disorder. The experiments were performed in accordance with the Declaration of Helsinki, and informed consent was obtained from all subjects.

\section{Stimuli and Procedure}

We used two versions of a task-cueing protocol inspired by the Wisconsin Card Sorting Test (Rubinstein et al., 2001; Milner, 1963) and adapted for measuring ERPs (Barcelo, 2003). Each trial consisted of a tonal cue followed by a target display with four key cards on top of one choice card, all centered on a computer screen 2 meters away from the observer (Figure 1A). The target 
stimulus subtended a visual angle of $3.5^{\circ} \times 3.5^{\circ}$, and remained on display until a response was given. Subjects were instructed to match the choice card with one of the four key cards following either two (color or shape; two-task condition) or three possible task rules (color, shape, or number; three-task condition). Subjects were told that the correct rule would change after a variable number of card sorts, and thus, they would have to shift their sorting rule accordingly. Before target onset, a tonal cue informed the subject whether to switch or to repeat the previous task $(200 \mathrm{msec}$ duration, $10 \mathrm{msec}$ rise/fall times; $75 \mathrm{~dB}$ SPL; $500 \mathrm{~Hz}$ and $1000 \mathrm{~Hz}$ binaural tones for "switch" and "repeat" cues, respectively; the tone-to-cue mapping was reversed for half of the subjects). Tonal switch cues occurred totally unpredictably in the two-task condition (switch probability $=.5$ ), or after two to five repeat trials in the three-task condition (switch probability $=$ 4). Subjects used their thumbs for responding while holding a four-button response panel in their palms. The far left button designated the key card on the far left of the display, the far right button designated the key card on the far right, and so on (Figure 1A). All the task sets declared in the instructions consisted of four-feature-stimulus to four-forced-response mappings $\left(\mathrm{S}_{i}-\mathrm{R}_{i}\right.$, with $i=1,2,3$, or 4 units). "Task set" denotes here, in a broad sense, a set of rules that govern the mapping between sensory inputs and motor responses (Braver et al., 2003). Responses $R_{1}$ and $R_{2}$ required pressing the two left buttons with the left thumb, and responses $R_{3}$ and $R_{4}$ involved pressing the two right buttons with the right thumb. For instance, when sorting by color, a "blue" target card was to be matched with the "blue" key card by pressing the rightmost response button $\left(S_{4}-R_{4}\right.$; Figure $\left.1 \mathrm{~A}\right)$. Response-to-cue intervals varied randomly between 800 and $1500 \mathrm{msec}$, with a constant cue-to-target onset asynchrony of $2250 \mathrm{msec}$ (Figure 1A). This task-cueing paradigm allowed us to segregate cue-locked (executive control) from target-locked (task execution) brain processes (Brass, Ullsperger, Knoesche, von Cramon, \& Phillips, 2005; Barcelo et al., 2002; Rubinstein et al., 2001).

These two task conditions were part of a longer series of experiments to explore the brain mechanisms of task-set switching (Barcelo, Periáñez, Corral, \& Escera, 2005; Periáñez, Nyhus, \& Barcelo, 2005) and were specifically designed to examine alternative accounts of task-novelty $\mathrm{P} 3$ activity in terms of involuntary orienting towards infrequent "oddball" cues, or to negative feedback signals (Dien, et al., 2004). In so doing, our threetask condition replicated the procedures used in our previous work (Periáñez et al., 2004; Barcelo et al., 2002), except for a higher probability of occurrence of the tonal switch cues. Moreover, both the task instructions and the structure of our three-task condition favored an appraisal of tonal cues as signals for future action rather than as feedback about past performance (Barcelo, 2003). This was strictly true in the two-task condition, where switch and repeat cues were presented totally at random and did not inform about the accuracy of the previous sort.

Prior to the experimental run, subjects were fully instructed about the rules in each task condition, and received practice for 5-10 min until they reached a criterion of $100 \%$ correct sorts during 1 minute. The order of presentation of each task condition was counterbalanced across participants. Each subject completed two blocks of 140 trials for each task condition, with a 10-min rest period between blocks. Overall accuracy was better than $85 \%$ correct trials in both tasks in all subjects. Here we report the brain potentials from correct trials only. Twenty-six trials with nonidentifiable novel sounds were randomly interspersed in one of the two blocks of trials in each condition. These 52 novel sounds were drawn from a larger set used in previous studies by Escera et al. (2003). Novel sounds appeared randomly between 700 and 1100 msec after a "repeat" task cue while keeping a constant novel-totarget stimulus onset asynchrony of $2250 \mathrm{msec}$ as in all other trials (Figure 1A). Novel sounds were not presented during the practice sessions, and each sound was used only once in the whole study. Task-repeat trials containing a novel sound were used to compute the "stimulus-novelty P3" waveforms, but were excluded from the computations of the cue-locked averages. The order of presentation of the trial block containing novel sound distracters was counterbalanced across subjects.

\section{ERP Data Acquisition and Analysis}

The electroencephalogram (EEG) was recorded from 28 tin electrodes positioned at Fp1, Fp2, F7, F3, Fz, F4, F8, FC1, FC2, FT3, FT4, T5, T3, C3, Cz, C4, T4, T6, P3, Pz, P4, TP3, CP1, CP2, TP4, M2, O1 and O2, and referenced to the left mastoid. The EEG signal was amplified (band pass, 1-30 Hz; $12 \mathrm{~dB} /$ octave roll/off), digitized at $500 \mathrm{~Hz} /$ channel and stored for off-line averaging. Electrode impedances were kept below $5 \mathrm{k} \Omega$. The electrooculogram (EOG) was recorded for eye blink correction. Epochs with EEG exceeding $\pm 75 \mu \mathrm{V}$ in amplitude or containing muscle artifacts were discarded. ERPs were obtained from correct trials only (see definition below). The averaging window was $700 \mathrm{msec}$ for auditory cues, novel sounds, and visual targets, including a 100-msec prestimulus baseline (Figure 1A). Individual ERP waveforms contained a minimum of 40 clean EEG epochs (range 45-98). ${ }^{3}$ A linked-mastoid reference was obtained off-line for the individual averages. Mean ERP amplitude values were measured relative to the 100-msec prestimulus baseline for the anterior aspect of the novelty P3 (330-350 $\mathrm{msec}$ poststimulus onset), its posterior aspect (500-520 msec), the N1 
(100-120 msec), the P2 (175-195 msec), and the N2 (240-260 msec) components. The anterior and posterior aspects of novelty P3 most likely reflect functionally distinct processes (Barcelo et al., 2002; Friedman et al., 2001). However, unless otherwise stated, the terms "task-novelty P3" and "stimulus-novelty P3" will denote the anterior aspect of this potential (330-350 msec; Figure 1C). The time window for measuring novelty P3 to the novel sounds was centered on its peak amplitude at Fz (i.e., $344 \mathrm{msec}$; Figure 1C). Latency and peak amplitudes for all components, as well as peak-to-peak N2-P3 amplitudes, were also computed. Target-locked P3b amplitudes (500-520 msec) were also computed to examine the implications of the experimental manipulations for the context updating model of the P300 component (Donchin \& Coles, 1988). Because target-locked ERPs did not elicit reliable novelty P3 activity, these results will be discussed in detail elsewhere (Barcelo et al., 2005). The "task-novelty" hypothesis was tested by comparing the novelty $\mathrm{P} 3$ responses to novel sounds and tonal switch cues ${ }^{4}$ in an ANOVA design with sound event (three-task switch cues, twotask switch cues, novel sounds), and electrode (Fz, Cz, $\mathrm{Pz}$ ) as the repeated measures factors. Normalized amplitudes from an array of 23 active electrodes were also employed for testing the scalp distribution of novelty P3 potentials to these auditory events (McCarthy \& Wood, 1985), with the following statistical design: Sound event (three-task switch cues, two-task switch cues, novel sounds) $\times$ Electrode $(\mathrm{Fp} 1, \mathrm{~F} 7, \mathrm{~F} 3, \mathrm{Fz}, \mathrm{F} 4, \mathrm{~F} 8$, Fp2, FT3, T3, C3, Cz, C4, T4, FT4, T5, TP3, P3, Pz, P4, TP4, T6, $\mathrm{O} 1$, and $\mathrm{O} 2)$. The task-novelty hypothesis predicted the absence of an interaction between sound event (threetask switch cues, two-task switch cues, novels) and electrode location for novelty P3 measurement (McCarthy \& Wood, 1985; Picton et al., 1978).

Temporal principal component analysis (PCA) has widely been used as an effective data reduction method for multivariate ERP datasets (Kayser \& Tenke, 2003). A temporal PCA was performed in order to parse away temporally overlapping processes in the ERP waveforms that might be influencing the topography of the tasknovelty $\mathrm{P} 3$ response to the auditory cues. The temporal epoch for this PCA ranged from a 100-msec prestimulus to a $600-\mathrm{msec}$ poststimulus onset. The variables in this temporal PCA consisted of 200 sample points (from -100 to $600 \mathrm{msec}$ ), representing the data matrix columns. The data matrix rows consisted of 2688 cases resulting from the product of 16 subjects, 2 task conditions (three tasks, two tasks), 3 types of auditory stimuli (novel sounds, switch cues, repeat cues), and 28 electrode sites. Individual ERP waveforms were lowpass filtered (cutoff $12 \mathrm{~Hz}, 24 \mathrm{~dB} /$ octave roll/off) before entering this analysis. Following previous reports, the PCA procedures adopted an unrestricted extraction of factors and were based on the covariance matrix in order to maintain polarity in the dataset (Kayser \&
Tenke, 2003). A Varimax rotation with the Kaiser correction was used to rotate the results to simple structure. A Promax solution was also computed, although it was largely consistent with the Varimax solution and will not be commented further in the Results section. The SPSS v11.0 statistical software was used for all PCA computations. After obtaining the factor solution, we reconstructed the portion of the grand averages accounted for by each factor by multiplying the factor loadings for each time point by the mean and standard deviation of the factor scores for the corresponding task condition. We then tested the "task novelty" hypothesis on the resulting PCA factor scores (Figure 3A and B) using the ANOVA designs described above. Greenhouse-Geisser epsilon ( $\boldsymbol{\varepsilon}$ ) correction was used to evaluate $F$ ratios for within-subject effects involving more than two degrees of freedom. Significant interactions involving electrode site were examined through simple tests of effects at each scalp site to locate the source of the interaction. Any significant effects involving the interaction between sound event and electrode were evaluated after scaling the amplitudes for each task by the vector amplitude measured across electrodes in each participant (McCarthy \& Wood, 1985).

Behavioral switch costs were obtained from both correct (reaction times [RTs]) and failed trials (error rates). Failed trials were defined as those where (a) the subject did not follow the instruction cue to switch or to repeat the previous task, or (b) they failed to select the correct response within the currently active task set (Barcelo, 2003). Reaction times and errors were subjected to a main ANOVA with block (with/without novels), task (three task, two tasks) and trial cueing (switch, repeat) as the repeated measures factors. Another ANOVA design focused on target responses following each auditory event, with factors task (three tasks, two tasks) and trial (switch, repeat, novel). All post hoc tests of simple effects were performed using the Bonferroni correction with a significance level of $p<.05$. Finally, the association between behavioral performance and mean novelty P3 amplitudes at Fz, $\mathrm{Cz}$, and $\mathrm{Pz}$ sites was assessed with a series of Pearson product-moment correlations with a significance level of $p<.05$ (two-tailed).

\section{RESULTS \\ Behavioral Results}

The three- and two-task conditions did not differ in their overall error rates or mean response latencies $\left(F_{\mathrm{S}}<1\right)$. As compared with repeat trials, switch trials reduced the speed [mean RT \pm SEM for repeat: $1079 \pm 56 \mathrm{msec}$ vs. switch: $1117 \pm 60 \mathrm{msec} ; F(1,15)=6.8, p<.02]$ and accuracy of subjects' responses to the target cards [mean error rate $\pm S E M$ for repeat: $4.7 \pm 0.9 \%$ vs. switch: $8.8 \pm 1.5 \% ; F(1,15)=29.1, p<.0001]$. In blocks 
containing novel sounds, subjects were marginally slower [mean RT \pm SEM with novels: $1105 \pm 52 \mathrm{msec}$ vs. without novels: $1071 \pm 60 \mathrm{msec} ;(1,15)=3.1, p=.1]$ and scored more errors [mean error rate \pm SEM with novels: $8.5 \pm 1.4 \%$ vs. without novels: $6.0 \pm 1.3 \%$; $F(1,15)=4.5, p<.05]$ as compared with trial blocks free of novel distracters. More specifically, the subjects' responses to targets following the occurrence of a novel sound were slower [mean RT \pm SEM after a novel: $1198 \pm 80 \mathrm{msec}$ vs. all other trials: $1112 \pm$ 60 msec; $F(1,15)=8.6, p<.01]$ and less accurate [mean error rate \pm SEM after a novel: $10.9 \pm 2.0 \%$ vs. all other trials: $7.8 \pm 1.3 \% ; F(1,15)=7.5, p<.02$ ] relative to all other trials in the block. Thus, both novel sounds and switch cues impaired performance in the following trial, a result consistent with extensive behavioral data about the distractibility effects of contextual novelty (Escera et al., 1998, 2000) and with the well established task-switch costs in response speed and accuracy (Monsell, 2003; Rubinstein et al., 2001).

Behavioral switch costs differed between two-task and three-task conditions, whereas this was not the case for the behavioral impairments caused by the novel sounds. Figure $1 \mathrm{~B}$ shows that mean RTs after a novel sound did not differ across task conditions $(F<1)$. In turn, task-switch costs in RT (RTs in switch-repeat trials) differed between both task conditions [mean RT cost \pm SEM for the three-task condition: $120 \pm 33 \mathrm{msec}$ vs. the two-task condition: $35 \pm 19$ msec; $F(1,15)=4.8, p<$ .05 ; see Figure $1 \mathrm{~B}]$. Likewise, the response accuracy after a novel distracter did not vary as a function of the task condition $(F<1)$, whereas task-switch costs in response accuracy (\% errors in switch-repeat trials) were larger in three-task compared with two-task conditions [\% errors \pm SEM for the three-task condition: $7.3 \pm 2.3 \%$ vs. the two-task condition: $0.9 \pm 1.2$ msec; $F(1,15)=$ $9.7, p<.01]$.

\section{ERP Results}

Averaged brain responses to tonal task cues and novel sounds are shown in Figure 1C. Novel sounds elicited similar brain potentials in both task conditions, and data from all novel sounds were pooled into one single ERP average per subject. The grand-average stimulusnovelty P3 potential showed its usual peak latency (344 msec at Fz; Figure 1C) and frontal-central distribution (Figure 1D). Task-switch cues in the threeand two-task conditions elicited brain P3 potentials with similar latency, morphology, and amplitude over midline regions (interaction of Sound event $\times$ Electrode, $F<1$ ). Despite this, planned comparisons revealed marginally larger task-novelty P3 amplitudes to switch cues in the three-task condition compared to the two-task condition at $\mathrm{Cz}$ and $\mathrm{Pz}(p s<.1)$, but not at Fz $(p=.22)$. Compared to task-switch cues, task-repeat cues elicited much reduced $\mathrm{P} 3$ potentials over midline sites in both the three-task and two-task conditions $[F(1,15)=12.6$, $p<.003$, for the main trial cueing effect; see Figure 1C]. Planned contrasts revealed that differences were mainly found at $\mathrm{Cz}(p s<.02)$ and $\mathrm{Pz}(p s<.006)$ sites, rather than at $\mathrm{Fz}(p \mathrm{~s}>.12)$, even though the third-order interaction between task, cueing event, and electrode did not reach significance $[F(1,15)=1.1, p=.32]$. These differences were not caused by a discrepancy in earlier N1 activity between the two types of cueing events ( $p=.13$; see Figure $1 \mathrm{C}$ ). Hence, only the more reliable $\mathrm{P} 3$ responses to the task-switch cues were considered in the comparisons with stimulus-novelty P3 activity.

Task-novelty $\mathrm{P} 3$ responses to the switch cues were comparatively smaller in amplitude than those evoked by the novel sounds over frontal $(p<.004)$, but not parietal, regions $(F \mathrm{~S}<1)$. This resulted in distinct scalp topographies for stimulus-novelty $\mathrm{P} 3$ and task-novelty P3 [Sound event $\times$ Electrode interaction, $F(44,660)=$ 15.0, $p<.0001]$. These differences did not translate into corresponding variations in peak-to-peak N2-P3 amplitude $\left(F_{S}<1\right.$; Figure $\left.1 \mathrm{C}\right)$. These results, together with a series of analyses of the scalp topography of cue-locked brain activity, suggested that the task cuesbut not the novel sounds-were associated with a frontally distributed, sustained negative field potential already present as early as $100 \mathrm{msec}$ postcue onset, and extending to encompass the novelty $\mathrm{P} 3$ latency window (see Figure 1C and D). Finally, target P3b potentials showed its usual maximum over mid-parietal regions. However, mean $\mathrm{P} 3 \mathrm{~b}$ amplitudes to the visual targets were not sensitive to the experimental manipulations in task-set (two vs. three tasks; $F<1$ ), nor to contextual information (switch vs. repeat trials; $F<1$ ) (see Figure 2).

\section{Covariance and Principal Component Analyses}

Two different analytical strategies suggested that a frontally distributed and sustained negative field potential (peak latency $110 \mathrm{msec}$ at Fz; N1 in Figure 1C) extended over the recording epoch, thus overlapping and distorting concurrent P3 activity to the task cues. Firstly, when mean N1 amplitudes were introduced as covariates in the analyses of novelty $\mathrm{P} 3$ potentials, the scalp topography of thus corrected $\mathrm{P} 3$ voltages did not differ between novel sounds and switch cues [Sound event $\times$ Electrode interaction, $F(44,660)=1.6, p=.2$; Figure $1 \mathrm{D}$, bottom] . This correction effectively removed the contribution of the frontal negative field potential associated with the task-switch cues. ${ }^{5}$ Secondly, when PCA was employed to extract the orthogonal component structure of the brain potentials to novel sounds and tonal switch cues, four Varimax-rotated factors explained over 94\% of the variance in the data matrix. These four factors reflected 


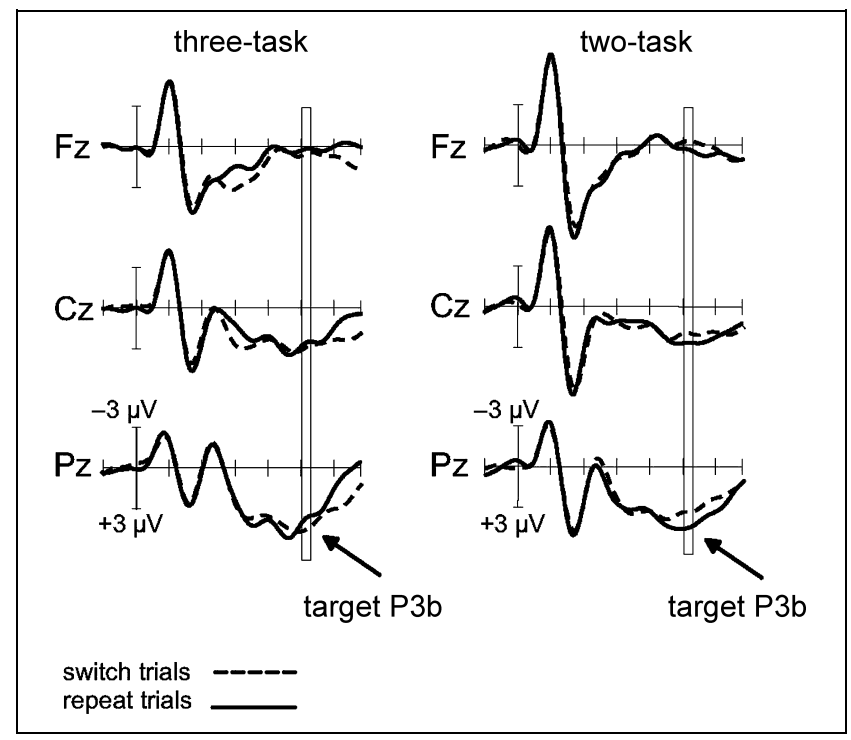

Figure 2. Grand ERP averages to visual targets. The target $\mathrm{P} 3 \mathrm{~b}$ component is shown at three midline electrodes in response to task-switch (broken line) and task-repeat (solid line) visual targets in the three-task and two-task conditions.

the main peaks and troughs in the waveforms, and were named after the peak latencies of their factor loadings: F520 (57\% of explained variance in the waveforms), F330 (16\%), F180 (14\%), and F110 (7\%), respectively (Figure 3A). The timing and scalp distribution of factor scores F110 and F330 corresponded with those of the fronto-central N1 and novelty $\mathrm{P} 3$ potentials, respectively (Figure 3A). Although factor loadings for the F110 component peaked $110 \mathrm{msec}$ postcue onset, this factor still showed sustained activation extending from 250 to 450 msec postcue onset. Figure 2B shows the normalized voltage maps of factor scores F110 and F330 in the latency window of 330-350 msec poststimulus onset for novel sounds and task-switch cues in the two-task and three-task conditions. The scalp topography of normalized F330 scores did not differ between novel sounds and tonal switch cues [Sound event $\times$ Electrode interaction, $F(44,660)=1.5, p=.3]$. In turn, the scalp distribution of normalized F110 scores differed significantly between novel sounds and switch cues in the latency range 330-350 msec postcue onset $[F(44,660)=$ $12.6, p<.0001$; Figure $3 \mathrm{~B}]$. Finally, the scalp topographies of factor scores F110 and F330 for the switch cues did not differ between the three-task and two-task conditions ( $F \mathrm{~s}<1$; Figure 3B). Prior to normalization, a comparison of F330 values for the three sound events revealed larger F330 amplitudes to the novel sounds as compared to the other two task-switch cues at Fz but not at $\mathrm{Cz}$ and Pz sites [Sound event $\times$ Electrode interaction, $F(4,12)=6.4, p=.005$; mean voltage at $\mathrm{Fz}$ for novels $=0.76 \mu \mathrm{V}$; three-task cues $=-0.17 \mu \mathrm{V}$; twotask cues $=-0.17 \mu \mathrm{V}$ ]. In turn, the intensity of F110 factor scores in response to switch cues did not differ at midline sites between the two-task and three-task conditions ( $F$ S $<1$; Figure 3B).

\section{Correlation Analyses}

In order to assess further whether these two concurrent processes, the sustained frontal negativity and the novelty P3, were related to any specific task-set switching mechanism, we examined their association with

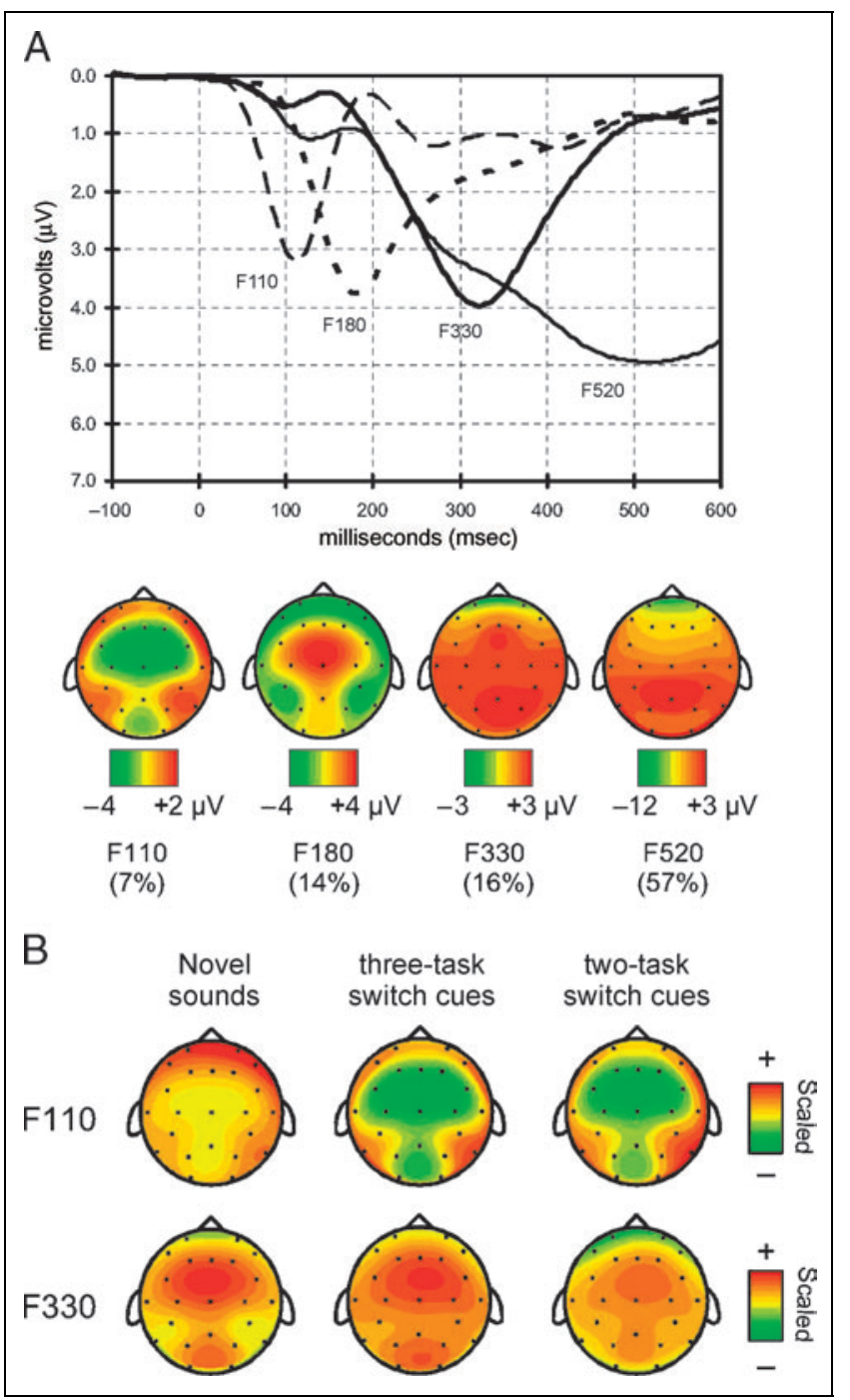

Figure 3. Principal component analysis (PCA). (A) Temporal dynamics of factor loadings (top) and scalp topographies of factor scores (bottom) for the first four PCA components (Kayser \& Tenke, 2003). Factors were labeled using the peak latency of their loadings (top). The scalp topography of the factor scores are ordered chronologically (bottom, nose upwards). The percentage of accounted variance for each factor is given in parentheses. (B) Spline-interpolated scalp maps of brain responses to novel sounds, two- and three-task switch cues for factor scores of F110 (upper row) and F330 (lower row). The time window for averaging brain activity in all maps was 330-350 msec. Novel sounds and tonal switch cues yielded equivalent scalp topographies for F330 factor scores (bottom). 
behavioral switch costs. We reasoned that if these two brain potentials were to reflect executive control of task-set switching, then their magnitude should be related to the speed and efficiency of responses to the target cards. Consistent with this prediction, individuals with the largest $\mathrm{P} 3$ amplitudes to the switch cues showed the least mean switch costs (switch minus repetition trials) in their RTs to the target cards. Negative correlations were strongest for $\mathrm{P} 3$ amplitudes measured at the vertex $(r=-.55, p<.03$, two-tailed $)$ and mid-parietal region $(r=-.70, p<.003$, two-tailed). Conversely, a distinct pattern of negative correlations was observed between the peak amplitude of the N1 potential to the task cues at mid-frontal sites, and the mean number of errors during both switch $(r=-.60$, $p<.02$, two-tailed) and repetition trials $(r=-.52$, $p<.04$, two-tailed). In contrast, the mean amplitude of stimulus-novelty P3 did not show any pattern of significant correlations with behavioral responses to the first target after a novel. This was the case both when correlations between novelty P3 and behavior were computed separately for two-task and three-task conditions and when they were computed on data collapsed across both task conditions.

\section{DISCUSSION}

The present results suggest that when a familiar event announces a change in task, it activates a neural network involved in novelty processing and disrupts behavior in a similar way as novel distracters do (cf., Barcelo et al., 2002). Nontarget events with regularity and physical properties similar to our task-switch cues, but delivered in single task-set paradigms (i.e., "oddball" tasks), fail to elicit reliable novelty P3 activity (Courchesne, Hillyard, \& Galambos, 1975; Squires, Squires, \& Hillyard, 1975). This became apparent from the brain responses to our task-repeat cues, which ceased to evoke novelty P3 activity as the same task was gradually rehearsed (Kopp, Tabeling, Moschner, \& Wessel, in press; Barcelo et al., 2002). Moreover, both novel sounds and task-switch cues impaired performance in the following trial, a result consistent with the distractibility effects of stimulus novelty (Escera et al., 1998, 2000) and with the well established task-switch costs in response speed and accuracy (Monsell, 2003). These results lend support to our task novelty hypothesis that appraisal of novel tasks and novel events both engage a common neural network for processing contextual novelty. Next, we discuss the observed similarities and disparities between stimulus-novelty P3 and task-novelty P3 activity in line with current models about the control of task-set switching operations (Koechlin et al., 2003; Monsell, 2003; Barcelo et al., 2002), and in the face of alternative accounts such as stimulus novelty (Friedman et al., 2001), "oddball" effects (Dien et al., 2004; Polich, 2003), orienting to negative feed- back (Dien et al., 2004), and involuntary attentional shifting (see Dien et al., 2004 for a review; Escera et al., 1998, 2000).

\section{Task Switching Elicits Transient Novelty P3 Activity}

We found that a familiar, repetitive, and task-relevant event elicited novelty P3 activity whenever it conveyed contextual information about an impending change in task. The degree of activation in the novelty $\mathrm{P} 3$ network seemed to depend on the informational content of the eliciting stimulus for selecting the next target response. From an information theory approach (Deco \& Obradovic, 1996; Berlyne, 1957), task-switch cues in our three-task condition conveyed a larger amount of information (entropy) than those in the two-task condition because the former events were less probable and called for retrieval of a larger number of task sets from memory (Koechlin et al., 2003). In other words, task-switch cues generated larger response uncertainty and conflict in three-task than in two-task conditions (Berlyne, 1957). This interpretation is consistent with results from a previous study, where the most informative task cues elicited the largest task-novelty P3 potentials (Barcelo et al., 2002). This analysis is also consistent with the extant literature in that the more infrequent and unexpected the event, the larger the novelty P3 potentials (Polich, 2003; Friedman et al., 2001; Courchesne et al., 1975). Indeed, the rarest and most novel of all sounds conveyed the greatest amount of information (entropy) in our task-cueing paradigm. ${ }^{6}$ Nevertheless, stimulus probability alone cannot account for the distinct task-novelty P3 responses in our two-task condition, where switch cues $(p=.5)$ elicited larger novelty $\mathrm{P} 3$ s than the equally probable repeat cues $(p=.5)$. The information conveyed by the switch cues about an imminent change in task, rather than their overall probability of occurrence, is to be held responsible for these modulations in tasknovelty P3 activity (Monsell, 2003; Rubinstein et al., 2001). The information content of our task cues depended not only on their probability of occurrence but also on their learned association with task-set and contextual information (Koechlin et al., 2003). ${ }^{6}$ When a task cue was repeatedly associated with a "no-change" in task, as with a monotonous succession of task-repeat cues, then activation in the novelty P3 network weakened or ceased completely (Barcelo et al., 2002). These modulations in novelty P3 activity do not seem to be caused simply by the salience or task relevance of cueing events, as suggested by the absence of differences in early N1 responses to switch versus repeat cues (Näätänen, 1990). In sum, task-novelty P3 may not be merely explained as a novelty response to nonidentifiable events (Courchesne et al., 1975), nor as an "oddball" response to rare events (Dien et al., 2004; Squires et al., 1975). In contrast, current models of task switching, combined 
with an information theory approach, may account for the observed changes in novelty P3 activity to highly unexpected, novel task-irrelevant distracters (Courchesne et al., 1975; Squires et al., 1975), as well as to familiar, repetitive, and task-relevant events that announce a change in mental set to a new task set (Koechlin et al., 2003; Barcelo et al., 2002).

Task-novelty P3 responses to the switch cues do not merely reflect a response to the negative feedback (i.e., to evaluation of an erroneous past action; cf., Dien et al., 2004). In this and in previous studies, task-switch cues were defined as signals for future action (Barcelo et al., 2002). This was strictly true in the two-task condition, where delivery of switch and repeat cues was independent of the subject's accuracy in the previous trial. Even so, two-task switch cues elicited reliable task-novelty P3 activity that predicted the behavior to the next target card. This is exactly what one would expect for a mechanism involved in anticipatory task-set switching operations (Monsell, 2003; Barcelo et al., 2002), as switch cues conveyed meaningful contextual information towards the selection of the next target response. Our task-switch cues prompted for an update of the active task set in working memory, although they explicitly informed neither about the next correct task set nor about the exact response selection within a particular task set. This probably explains a rather modest association of task-novelty P3 activity with behavioral switch costs (Nicholson, Karayanidis, Poboka, Heathcote, \& Michie, 2005; Barcelo et al., 2002). A more comprehensive neurocognitive model of behavioral switch costs needs to consider other sources of cue-related as well as target-related brain activations. In contrast, stimulus-novelty P3 amplitudes were unrelated to the behavioral responses to the following target. In fact, we have not been able to find any such correlations in an extensive review of the literature on behavioral distraction using oddball tasks (Friedman et al., 2001; Escera et al., 1998, 2000). This is consistent with our finding that novel sounds elicited similar novelty $\mathrm{P} 3$ responses and led to comparable behavioral impairments in two-task and three-task conditions (Figure $1 \mathrm{~B}$ ). All these evidences add up to our proposal that novel distracters were not part of any of the task sets explicitly defined in the instructions (each with an $\mathrm{S}_{i}-\mathrm{R}_{i}$ mapping, where $i=1,2,3$, or 4 units). Instead, novel sound distracters implicitly afforded a new "dummy" task set $\left(\mathrm{S}_{x}-\mathrm{R}_{0}\right)$, where any nonidentifiable sound features $\left(\mathrm{S}_{x}\right)$ were implicitly linked to a nonresponse $\left(\mathrm{R}_{0}\right)$ motor program. ${ }^{6}$ This would explain the typical absence of association between stimulus-novelty P3 and the behavior to the next target because a novel distracter affords a switch to a different task set than the one ruling the subject's overt behavior. Under this assumption, the onset of a novel sound distracter not only generates a large amount of entropy in the neural system controlling the selection of the next response (Koechlin, et al., 2003) but also affords a switch to a new "silent" task set (Corbetta \& Shulman, 2002; Escera et al., 1998).

To sum up, task-novelty P3 activity cannot be explained as an orienting reaction to negative feedback. In turn, the task novelty hypothesis predicts novelty P3 activity to both positive and negative feedback signals (Butterfield \& Mangels, 2003; Nieuwenhuis et al., 2002) in so far as both are informative events that may call for an update in the active S-R mapping in order to adjust it to anticipated changes in task demands (but see Kopp et al., 2006, for an alternative view). This proposal would also be consistent with the interpretation of novelty P3 activity in a pioneer work using a WCSTanalogue as a feedforward mechanism "to modify the expectancies for future perceptual or motor activity" (Stuss \& Picton, 1978).

\section{Task-novelty $\mathbf{P} 3$ Coexists with a Sustained Frontal Negativity}

Despite the similarities disclosed here, stimulus-novelty P3 and task-novelty P3 were also differently modulated by the present task conditions (Figure 1C and D). Tasknovelty P3-but not stimulus-novelty P3-was modulated by task-set information such as the rate of task switching and the number of task sets held in memory (Figure 1C; Periáñez et al., 2005; Barcelo et al., 2002). More importantly, a frontally distributed slow negative field potential was specifically associated with the task cues, but was absent from the brain responses to outof-set novel distracters. The mechanism responsible for this negativity was already active $100 \mathrm{msec}$ postcue onset (Figure 1C) and, according to our PCA results, it remained active during the latency window of novelty P3 (Figure 3A and B). This finding concurs with an interpretation of this frontal negativity as a sustained brain activation that coexists with transient novelty P3 activity during task switching (cf., Braver, et al., 2003), and is also consistent with recent evidence that the prefrontal cortex precedes the posterior association cortices in the temporal organization of cognitive control (Brass et al., 2005; Periáñez et al., 2004). The early timing of this frontal negativity, contemporaneous with other preattentive change-detection mechanisms in auditory cortex (i.e., N1 and mismatch negativity [MMN]), was not predicted by current models of selective attention (Hillyard et al., 1995; Näätänen, 1990). As we did not have any explicit hypotheses about this frontal negativity, we can only make post hoc inferences about its functional role based on evidence from this and other recent studies.

Slow negative field potentials with similar frontal scalp distribution have been reported in several taskswitching studies (Brass et al., 2005; Nicholson et al., 2005; Rushworth et al., 2002). These frontal negativities have been typically described as slow-wave deflections towards the end of the cue-locked period, and may even 
extend over to the target period (Nicholson et al., 2005; Rushworth et al., 2002). We have observed similar negative slow waves over frontal scalp regions in our previous work (cf., Barcelo, Muñoz-Céspedes, Pozo, \& Rubia, 2000, Figure 5; Barcelo et al., 2002, Figures 2 and 3). Two key features of this negativity are worth mentioning here: (a) it was associated only with those auditory events that had been explicitly linked to the active task sets; (b) similar N1 amplitudes and F110 scores were recorded to both switch and repeat cues in the three-task and two-task conditions (Figures 1C and $3 \mathrm{~B}$ ). This was a critical finding that carries two further corollaries. First, an enhanced fronto-central N1 response to all task cues suggests that these were tagged as task-relevant events at an early processing stage and were consciously perceived (Näätänen, 1990). Hence, it is questionable that the neural decision about whether to trigger the ensuing novelty $\mathrm{P} 3$ can be dubbed as "automatic" or "involuntary" (cf., Sergent, Baillet, \& Dehaene, 2005). Second, the frontal negativity is unlikely to index a specific task-set switching mechanism (such as uploading of a new task-set on active memory). Instead, this sustained frontal activation might reflect active maintenance of task-set information over trials (Braver et al., 2003; Koechlin et al., 2003), consistent with the "processing negativities" of attentional-trace models (Näätänen, 1990), but also with the monitoring of response conflicts among competing task sets (Carter, et al., 1998). The present ERP data are compatible with any of these alternatives, and further research will be needed to decide among them.

Finally, although this frontal negativity might explain the enhanced N1 to task cues, it was not a prerequisite for novelty P3 elicitation. Two change-detector mechanisms, $\mathrm{N} 1$ and MMN, have been proposed to trigger novelty P3 activity in oddball-like tasks (Näätänen, 1990) whenever "some momentary threshold is exceeded" (p. 231). Following our rationale, this threshold might be expressed as the amount of information (response entropy) conveyed by a signal (Koechlin et al., 2003). Another straightforward prediction from Näätänen's (1990) model is that task-set information indexed by the frontal ("processing") negativity should not interact with the preattentive neural mechanisms involved in MMN generation. This prediction could be easily tested by interspersing a monotonous background of task-irrelevant "standard" tones for comparison with both novel sounds and taskcueing events in our task-switching paradigm.

\section{Task Switching versus Single Task-set Paradigms in Attention Research}

From a methodological point of view, our results indicate that dual-task paradigms may be better suited than single task-set paradigms for exploring the dynamic interplay between endogenous and exogenous factors controlling human cognition. The vast majority of stud- ies on the neural bases of human attention have employed single task-set paradigms such as "oddball" tasks (Näätänen, 1990; Donchin \& Coles, 1988) or selectiveattention tasks (Posner, 2004; Hillyard et al., 1995). In these settings, the onset of a novel distracter (i.e., a sudden bell ring, a flash of light) elicits novelty $\mathrm{P} 3 \mathrm{ac}-$ tivity that is often taken to reflect an involuntary shift in attention towards the novel distracter and away from the main task. Accordingly, the neural workings of novelty P3 are often described as stimulus-driven or automatically generated (Polich, 2003; Friedman et al., 2001; Escera et al., 1998). The present results call for a revision of this view. Our familiar and repetitive tonal cues prompted for intentional switches and repetitions in the ongoing task set, and were tagged as task-relevant events at an early stage of neural processing, as indicated by an enhanced frontal-central N1 response (Näätänen, 1990). Yet, only those cues that called for an intentional switch in task elicited reliable novelty P3 activity. Our finding is consistent with $\mathrm{P} 3$ results from a recent study on the attentional blink phenomenon, where participants performed fast attentional switches from task1 to task2 in order to assess their inattention to events in task2 (Sergent et al., 2005). These authors reported frontally distributed P3a activity (novelty P3) to those events that were perceived during the attentional blink, but not to those events that did not reach consciousness. Hence, awareness of events in task 2 and elicitation of novelty P3 were perfectly correlated with the subjects' ability to switch tasks successfully (Sergent et al., 2005). This finding concurs with our proposal that taskset switching is one antecedent condition for elicitation of novelty P3 activity (cf., Ranganath \& Rainier, 2003). Likewise, it suggests that dual-task paradigms may offer new insights into the level of automaticity of attentional mechanisms traditionally explored under single task-set conditions (cf., Posner, 2004; Näätänen, 1990).

Single task-set paradigms, such as oddball tasks, make an emphasis on the goal-directed control of task-set maintenance operations. By definition, these tasks were not designed to examine task-set switching operations. The interpretative problems crop up when oddball-like tasks are employed to explore the stimulus-driven control of attention. Novel distracters delivered in single task-set paradigms may be implicitly linked to a "no-go" response resulting in a "silent" task set (task0; $\mathrm{S}_{x}-\mathrm{R}_{0}$ ), different from the main task sets explicitly declared with the task instructions (task1, task2, etc.). In such cases, the onset of a novel deviant will prompt for a switch from task1 to task0 and back to task1, and the brain responses to the novels will thus reflect a mixture of stimulus-driven and goal-directed task-set switching operations. This proposal is consistent with accepted views of novelty $\mathrm{P} 3$ as an orienting response to the novels (Escera et al., 1998; Näätänen, 1990). An adequate control for the brain responses to novel distracters in oddball tasks should consider the brain responses to 
familiar task-relevant cues signaling either to intentionally switch or repeat the ongoing task set. Without such a control, single task-set paradigms may be seriously overlooking or misinterpreting the goal-directed control of task-set switching operations (Barcelo, 2003; Yantis, 2000). For these reasons, the present results reveal more top-down control in the brain responses to novel distracters than previously suspected (Escera et al., 2003; Sussman et al., 2003; Pashler et al., 2001). From this new perspective, single task-set paradigms, which employ attention-capturing singletons like novel distracters or light flashes (Posner, 2004; Polich, 2003; Friedman et al., 2001), may be seen as a special class of task-switching paradigms, where participants are intermittently prompted to switch their mental set from one main task (task1; "respond to right-field bars") to a secondary dummy task (task0; "stop on the novels"). This proposal carries crucial implications for a reinterpretation of novelty P3 activity in line with current models about the cognitive control of human behavior.

\section{Implications for Current Models of Attentional Control}

\section{Towards a Theoretical Integration}

The similarity disclosed here between task-novelty P3 and stimulus-novelty $\mathrm{P} 3$ calls for an integration of results from task switching and behavioral distraction studies. Novelty oddball tasks may be seen as a special class of task-switching paradigms, as changing tasks also normally involve some physical change in stimulation. Conversely, the onset of a novel distracter represents an external call for doing or thinking something different (cf., Barcelo et al., 2002), an idea that lies behind the use of flashing ads and banners in the media. The theoretical isolation of these two research programs could be attributed to their distinct definition of contextual novelty. The former perspective defines novelty in terms of a change in the internal representations of task-sets or stimulus-response mappings (the task context) (Brass \& von Cramon, 2004; Monsell, 2003). The latter view defines novelty as a rare change in physical stimulation against a repetitive stimulus background (the stimulus context) (Polich, 2003; Donchin \& Coles, 1988). A desirable integration between these two approaches could be achieved by emphasizing the intrinsic interplay between the exogenous (i.e., physical change) and endogenous (i.e., past knowledge and experience) aspects of contextual novelty (Pashler et al., 2001; Yantis, 2000). For instance, two physically identical whistle blows may either start or finish a football game, but their precise meaning for selecting our next action will depend on our recent memories. From an information theory approach, both exogenous and endogenous factors may contribute to the amount of information (entropy) conveyed by environmental events for response selection (Koechlin et al., 2003). Ac- cording to our view, the "momentary threshold" for novelty P3 elicitation would not depend solely on the "taskindependent" or "task-dependent" processing of sensory signals (Näätänen, 1990) but on the amount of entropy carried by these signals for selecting the next response (Koechlin et al., 2003).

\section{Novelty P3 Activity Reflects Cognitive Control}

The present results suggest that novelty P3 might be construed as the transient activation in a neural network involved in task-set switching (Barcelo et al., 2002), possibly reflecting part of the process through which an old task set is replaced by a new one for future goal-oriented behavior (cf., Ranganath \& Rainier, 2003). In our study, the call for novelty P3 elicitation seemed to depend on the information (entropy) of task events for response selection, over and above their relative frequency, familiarity, or task relevance. This interpretation is more general and can encompass traditional views of novelty $\mathrm{P} 3$ as an orienting response to taskirrelevant novel distracters (Friedman et al., 2001). Tasknovelty $\mathrm{P} 3$ responses were sensitive to the informational content of the cueing events, as derived from their relative frequency (Berlyne, 1957), but also by their previously learned associations with task-set (two vs. three tasks) and contextual (switch vs. repeat) information. An information theoretic analysis of the information conveyed by sensory events about future response selection can provide a formal account for the psychological concept of cognitive control (Koechlin et al., 2003; Deco \& Obradovic, 1996). From this perspective, novelty $\mathrm{P} 3$ responses could be seen as transient activations in a neural network involved in solving response uncertainty in the face of either a novel event or a new task context. The larger the novelty (entropy) conveyed by an environmental event, the larger the demands of control for response selection. Novelty P3 activity weakens when entropy is reduced, insofar as the system retrieves the information necessary to match the novel context with a suitable set of responses. This normally occurs when an appropriate $\mathrm{S}-\mathrm{R}$ mapping is updated in working memory. Novelty P3 is very sensitive to low probability events, as the relative frequency of occurrence is an important-although not the only-determinant of the information tradeoff associated with the strategic and goal-directed control of behavior (Koechlin et al., 2003; Huettel, Mack, \& McCarthy, 2002). This new account might explain novelty P3 activity to novel stimuli (Friedman et al., 2001; Courchesne et al., 1975), infrequent deviants (Polich, 2003; Squires et al., 1975), feedback signals (Butterfield \& Mangels, 2003; Stuss \& Picton, 1978), "no-go" signals (Periáñez et al., 2005; Rushworth et al., 2004), and first trial effects (Periáñez et al., 2005; Huettel et al., 2002). In line with our proposal, all these situations require the updating of multimodal task-set representations 
in working memory in the face of changing task demands. Further research will be needed to clarify whether novelty P3 activity in these situations indexes merely the selection, updating, and activation of a new task set (Barcelo et al., 2002), or also the interruption (Corbetta \& Shulman, 2002), inhibition, and deactivation of a previously active task set (Periáñez et al., 2005; Brass \& von Cramon, 2004; Monsell, 2003).

\section{The "Context Updating" Model of P300 Revisited}

The present results bring us back to the seminal papers by Courchesne et al. (1975) and Squires et al. (1975), who set out to solve the apparent paradox that P300-like potentials could be elicited by both attended and ignored events (i.e., "by unpredictable stimulus shifts that are ignored and by those that are task-relevant and are being actively sought out"; Squires et al., 1975, p. 387). These authors solved the P300 riddle of their time by showing that P3-like potentials elicited by task-relevant events ("the target P3b") were functionally and anatomically distinct from the brain P3 potentials elicited by stimuli seemingly irrelevant to the ongoing task ("the novelty P3"). ${ }^{7}$ Here we have been able to go one step further in the functional segregation of these endogenous late positivities. First, our task-cueing paradigm allowed us to isolate cue-locked executive control from target-locked task execution processes (Barcelo, 2003; Rubinstein et al., 2001). Second, we showed that the updating of task-set and contextual information was indexed by the cue-locked novelty $\mathrm{P} 3$ rather than by the target-locked P3b responses (Figure 2; Periáñez et al., 2005; Barcelo et al., 2002). This finding unveils a likely confounding in the measurement of the P300 component, which typically reflects a mixture of task-set switching (novelty P3) and task execution (target P3b) processes in response to infrequent target events in oddball tasks (Polich, 2003; Donchin \& Coles, 1988). The undoing of this mix-up seems a prerequisite for solving a long-standing dialectic about the functional role of these late positivities (Donchin \& Coles, 1988). Finally, the functional role of the neural network for novelty P3 elicitation could be best explored by means of explicit task sets and well-defined $\mathrm{S}-\mathrm{R}$ mappings (i.e., $\mathrm{S}_{i}-\mathrm{R}_{i}$, with $i=1,2,3 \ldots$ units), rather than using nonidentifiable novel events implicitly linked to nonresponse motor programs (i.e., $\mathrm{S}_{x}-\mathrm{R}_{0}$ ). Infrequent and nonidentifiable novel events generate maximal response entropy and activation in the novelty P3 network. However, it is important to explore the lower threshold of response entropy below which an interrupt signal will cease to activate this system (Näätänen, 1990). The use of task-cueing paradigms with explicit task sets and welldefined S-R mappings opens up the possibility to manipulate response entropy, and searching for stronger correlations between novelty P3 activity and behavioral performance.

\section{Novelty P3 and the Attentional Bottleneck}

The behavioral impairments associated with novelty $\mathrm{P} 3$ elicitation point to this brain mechanism as one likely element in the central bottleneck posed by some models of human attention and performance (Pashler et al., 2001). This proposal is consistent with the observation that novelty $\mathrm{P} 3$ recruits activity in a widespread fronto-posterior cortical network for attentional control (Ranganath \& Rainier, 2003; Corbetta \& Shulman, 2002; Knight, 1996). What is updated during novelty P3 elicitation does not correspond with the sensory representation of the eliciting stimulus. Rather, novelty P3 seems to reflect updating of task-set information (including a new S-R mapping) necessary for goal-directed selection of the next response. In consequence, the neural mechanisms behind novelty $\mathrm{P} 3$ could be held responsible, at least in part, for the behavioral impairments caused by novel distracters and task-switch cues, and might thus constitute one key element in a limited-capacity system (Näätänen, 1990), or central bottleneck for attentional control (Pashler et al., 2001; Ruthruff, Pashler, \& Klaassen, 2001). Following our rationale, the attentional bottleneck might derive from a structural limitation of the frontal-parietal network to update and maintain two competing S-R mappings simultaneously active in working memory. The behavioral impairments might reflect the time taken to reconfigure this frontal-parietal network back and forth with a new task set, rather than any extra resources necessary for holding two or more task sets simultaneously in active memory. This idea meets with previously held views that an "attentional trace only exists while it is maintained by the executive mechanism" (Näätänen, 1990, p. 232). It is also plausible that a given task set may be gradually readjusted through learning and experience to incorporate in its S-R mapping a larger number of stimulus features (i.e., from $\mathrm{S}_{1}$ to $\mathrm{S}_{1}, \mathrm{~S}_{2}, \mathrm{~S}_{3} \ldots \mathrm{S}_{n}$ ), or/and a wider range of response alternatives (i.e., from $\mathrm{R}_{1}$ to $\mathrm{R}_{0}, \mathrm{R}_{1}, \mathrm{R}_{2}, \mathrm{R}_{3} \ldots \mathrm{R}_{n}$ ). This adaptive plasticity may account for strategic variations in behavioral efficiency (Braver et al., 2003; Huettel et al., 2002; Pashler et al., 2001), as well as for the attenuation of novelty $\mathrm{P} 3$ responses over successive repetitions of the same novel event (Barcelo et al., 2002; Courchesne et al., 1975). One final corollary of this new proposal is that novelty $\mathrm{P} 3$ activity should not be described as Pavlov's "what is it" orienting reaction (Courchesne et al., 1975), but rather as a more general "change your mind" mechanism of cognitive control at the core of human adaptive behavior.

\section{Summary and Conclusions}

Novelty P3 activity has been traditionally interpreted as an orienting response to rare or novel task-irrelevant distracters (Courchesne et al., 1975; Squires et al., 1975). In turn, the observed modulations in novelty $\mathrm{P} 3$ activity 
to task-switch cues could be interpreted as neural correlates of the internal reconfiguration or updating of goals, or the linking of sensory signals to their appropriate stimulus-response mappings (Rushworth et al., 2004; Braver et al., 2003), probably involving the recruitment of activation in lateral and medial prefrontal, as well as posterior, multimodal association cortices (Ranganath \& Rainier, 2003; Soltani \& Knight, 2000; Knight, 1996). The present results lend support and allow us to elaborate further on our original hypothesis that both novel distracters and familiar task-switch cues activate a common neural network for processing contextual novelty (Barcelo et al., 2002). Likewise, our interpretation of these results is consistent with an involvement of the novelty $\mathrm{P} 3$ neural network in a variety of cognitive tasks that demand a continual updating of task-set information (Ridderinkhof et al., 2004; Rushworth et al., 2004; Duncan \& Owen, 2000). This new proposal has the potential to explain frontal-central P3-like positivities recorded to deviant events, novel distracters, oddball targets, feedback signals, stop signals, and first trials, and could help us establish a theoretical bridge between apparently distinct processes such as task switching (Monsell, 2003), behavioral distraction (Sussman et al., 2003; Escera et al., 2000), and concept formation (Duncan \& Owen, 2000; Stuss \& Picton, 1978), or indeed, with many other situations where humans confront novelty (Shallice, 2002).

\section{Acknowledgments}

Supported by grants from the Conselleria d'Innovacio i Energia, Govern Balear PRIB 2004-10136 (to F. B.), the Spanish Ministerio de Educacion y Ciencia BSO2003-03885 (to F. B.), BSO2003002440 (to C. E.), and the Generalitat de Catalunya 2003XT00084, 2005SGR-00953 (to C. E.). We thank Bob Knight and three anonymous referees for their thoughtful comments and helpful suggestions. We also thank Etienne Koechlin for his help with the computations of the informational content of task events.

Reprint requests should be sent to Francisco Barcelo, Ed. Beatriu de Pinos, University of Illes Balears (UIB), Ctra Valldemossa km 7.5, Palma de Mallorca, Spain, or via e-mail: f.barcelo@uib.es.

\section{Notes}

1. The terms "task-novelty P3" and "stimulus-novelty P3" are used here as descriptive handles to denote the kind of operational procedures employed to elicit novelty P3 activity. According to the "task novelty hypothesis," these terms also denote a common explanatory construct because both taskswitch cues and novel distracters are proposed to activate the same neural network responsible for novelty P3 elicitation.

2. Note that our task cues are of a different nature from those used in the vast majority of studies on spatial or selective attention (Posner, 2004). Exogenous and endogenous cues in a Posner's paradigm orient attention within the same task set to a subset of all possible $\mathrm{S}-\mathrm{R}$ links already defined in the active $S_{i}-R_{i}$ mapping (i.e., $S_{\text {left field }}-R_{i}$ ). Typically, these studies were not designed to explore the neural mechanisms for switching to and reconfiguring a new attentional set (cf., Posner, 2004).

3. Similar brain responses were elicited by novel sounds in two-task and three-task conditions. Therefore, EEG data from all novel sounds were pooled into one single ERP average per subject.

4. The statistical design for testing the "task novelty" hypothesis considered P3 activity to the task-switch cues only. The first repeat cue following a switch in task also evoked tasknovelty P3 activity, although this attenuated quickly (Barcelo et al., 2002). The grand ERP averages for task-repeat cues did not show reliable task-novelty P3 activity (see Figure 1C), hence, were not considered in the present statistical design. The antecedent conditions for the differential attenuation of P3 responses to task-switch versus task-repeat cues have been addressed elsewhere (Barcelo et al., 2005).

5. Mean N1 rather than N2 amplitudes were used as covariates in this analysis for several reasons. First, distinct brain responses to novel sounds and task cues were already apparent at the N1 latency window, and the subsequent differences in N2 activity were partly due to this earlier frontal N1 effect. Second, this interpretation was consistent with results from the PCA, as the F110 factor remained active through 250-450 msec postcue onset, thus reflecting activation in the $\mathrm{N} 2$ latency range (Figure $3 \mathrm{~A}$ ). Third, novel sounds and taskswitch cues evoked similar peak-to-peak N2-P3 amplitudes at midline sites. Finally, the scalp topography of corrected P3-N1 amplitudes did not differ from that of the stimulus-novelty $\mathrm{P} 3$ (Figure 1D).

6. The overall probability of novel sound distracters was $p=.16$ in both two-task and three-task conditions. Because novel sounds were not related to the onset of the next target card, their information content can be formally estimated as $\mathrm{I}($ novel $)=\log _{2}\left(p(\right.$ novel, task 0$)-\log _{2}(p($ novel $) * p(\operatorname{task} 0)=$ $\log _{2}(0.16)-\log _{2}(0.16 * 0.16)=-\log _{2}(0.16)$. These computations assume that novel sounds were associated with a dummy task (where task0 is assumed to demand a "no-go" response; E. Koechlin, personal communication). On the other hand, the information conveyed by the switch cues depended not only on their overall probability of occurrence in the three-task $(p=.4)$ and two-task $(p=.5)$ conditions but more importantly, on their previously learned associations with task-set (two vs. three tasks) and contextual (switch vs. repeat) information (cf., Koechlin et al., 2003). In the three-task condition, sometimes the subject had to switch rule twice in a raw (i.e., switch1, switch2, reapeat1, etc.). In these cases, the information content was larger in switch1 than in switch2 trials (cf., Barcelo et al., 2002). In fact, our estimation of the information content of switch 2 cues in the three-task condition equals that of switch cues in the two-task condition ( $\sim 1$ bit; cf., Koechlin et al., 2003). Hence, the inclusion of switch2 trials in the ERP averages probably explains the marginal differences in novelty P3 amplitude between switch cues in the three-task and twotask conditions.

7. Although Courchesne et al. (1975) argued that their novelty $\mathrm{P} 3$ was probably a different phenomenon to the frontal P3a described by Squires et al. (1975), more recent high-density ERP mapping studies have shown that both components reflect varying degrees of activation in a common network of brain regions (Dien et al., 2004; Friedman et al., 2001).

\section{REFERENCES}

Barcelo, F. (2003). The Madrid Card Sorting Test (MCST): A task switching paradigm to study executive attention with event-related potentials. Brain Research Protocols, $11,27-37$. 
Barcelo, F., Muñoz-Céspedes, J. M., Pozo, M. A., \& Rubia, F. J. (2000). Attentional set shifting modulates the target $\mathrm{P} 3 \mathrm{~b}$ response in the Wisconsin card sorting test. Neuropsychologia, 38, 1342-1355.

Barcelo, F., Periáñez, J. A., Corral, M. J., \& Escera, C. (2005). Modulations of the endogenous $\mathrm{P} 3$ potential during task set switching and updating. Psychophysiology, 42, S34.

Barcelo, F., Periáñez, J. A., \& Knight, R. T. (2002). Think differently: A brain orienting response to task novelty. NeuroReport, 13, 1887-1892.

Berlyne, D. E. (1957). Uncertainty and conflict: A point of contact between information-theory and behavior-theory concepts. Psychological Review, 64, 329-339.

Brass, M., Ullsperger, M., Knoesche, T. R., von Cramon, D. Y., \& Phillips, N. A. (2005). Who comes first? The role of the prefrontal and parietal cortex in cognitive control. Journal of Cognitive Neuroscience, 17, 1367-1375.

Brass, M., \& von Cramon, D. Y. (2004). Decomposing components of task preparation with functional MRI. Journal of Cognitive Neuroscience, 16, 609-620.

Braver, T. S., Reynolds, J. R., \& Donaldson, D. I. (2003). Neural mechanisms of transient and sustained cognitive control during task switching. Neuron, 39, 713-726.

Butterfield, B., \& Mangels, J. A. (2003). Neural correlates of error detection and correction in a semantic retrieval task. Cognitive Brain Research, 17, 793-817.

Carter, C. S., Braver, T. S., Barch, D. M., Botvinick, M. M., Noll, D., \& Cohen, J. D. (1998). Anterior cingulate cortex, error detection, and the online monitoring of performance. Science, 280, 747-749.

Corbetta, M., \& Shulman, G. L. (2002). Control of goal-directed and stimulus-driven attention in the brain. Nature Reviews Neuroscience, 3, 201-215.

Courchesne, E., Hillyard, S. A., \& Galambos, R. (1975). Stimulus novelty, task relevance, and the visual evoked potential in man. Electroencephalography and Clinical Neurophysiology, 39, 3336-3338.

de Fockert, J., Rees, G., Frith, C., \& Lavie, N. (2004). Neural correlates of attentional capture in visual search. Journal of Cognitive Neuroscience, 16, 751-759.

Deco, G., \& Obradovic, D. (1996). An information-theoretic approach to neural computing. New York: Springer.

Dien, J., Spencer, K. M., \& Donchin, E. (2004). Parsing the late positive complex: Mental chronometry and the ERP components that inhabit the neighborhood of the P300. Psychophysiology, 41, 665-678.

Donchin, E., \& Coles, M. G. H. (1988). Is the P300 component a manifestation of context updating? Behavioral and Brain Sciences, 11, 343-356.

Duncan, J., \& Owen, A. M. (2000). Common regions of the human frontal lobe recruited by diverse cognitive demands. Trends in Neurosciences, 23, 475-483.

Escera, C., Alho, K., Schröger, E., \& Winkler, I. (2000). Involuntary attention and distractibility as evaluated with event-related brain potentials. Audiology and Neuro-Otology, 5, 151-166.

Escera, C., Alho, K., Winkler, I., \& Näätänen, R. (1998). Neural mechanisms of involuntary attention to acoustic novelty and change. Journal of Cognitive Neuroscience, 10, 590-604.

Escera, C., Yago, E., Corral, M. J., Corbera, S., \& Nunez, M. I. (2003). Attention capture by auditory significant stimuli: Semantic analysis follows attention switching. European Journal of Neuroscience, 18, 2408-2412.

Friedman, D., Cycowicz, Y. M., \& Gaeta, H. (2001). The novelty P3: An event-related brain potential (ERP) sign of the brain's evaluation of novelty. Neuroscience and Biobehavioral Reviews, 25, 355-373.
Hillyard, S. A., Mangun, G. R., Woldorff, M. G., \& Luck, S. J. (1995). Neural systems mediating selective attention. In M. S. Gazzaniga (Ed.), The cognitive neurosciences (pp. 665-681). Cambridge: MIT Press.

Huettel, S. A., Mack, P. B., \& McCarthy, G. (2002). Perceiving patterns in random series: Dynamic processing of sequence in prefrontal cortex. Nature Neuroscience, 5 , 485-490.

Kayser, J., \& Tenke, C. E. (2003). Optimizing PCA methodology for ERP component identification and measurement: Theoretical rationale and empirical evaluation. Clinical Neurophysiology, 114, 2307-2325.

Knight, R. T. (1996). Contribution of human hippocampal region to novelty detection. Nature, 383, 256-259.

Koechlin, E., Ody, C., \& Kouneiher, F. (2003). The architecture of cognitive control in the human prefrontal cortex. Science, 302, 1181-1185.

Kopp, B., Tabeling, S., Moschner, C., \& Wessel, K. (2006). Fractionating the neural mechanisms of cognitive control. Journal of Cognitive Neuroscience, 18, 949-965.

McCarthy, G., \& Wood, C. C. (1985). Scalp distributions of event-related potentials: An ambiguity associated with analysis of variance models. Electroencephalography and Clinical Neurophysiology, 62, 203-208.

Milner, B. (1963). Effects of different brain lesions on card sorting. Archives of Neurology, 9, 100-110.

Monsell, S. (2003). Task switching. Trends in Cognitive Sciences, 7, 134-140

Näätänen, R. (1990). The role of attention in auditory information processing as revealed by event-related potentials and other brain measures of cognitive function. Behavioral and Brain Sciences, 13, 201-288.

Nicholson, R., Karayanidis, F., Poboka, D., Heathcote, A., \& Michie, P. T. (2005). Electrophysiological correlates of anticipatory task-switching processes. Psychophysiology, $42,540-554$.

Nieuwenhuis, S., Ridderinkhof, K. R., Talsma, D., Coles, M. G., Holroyd, C. B., Kok, A., \& van der Molen, M. W. (2002). A computational account of altered error processing in older age: Dopamine and the error-related negativity. Cognitive, Affective, and Behavioral Neuroscience, 2, 19-36.

Nobre, A. C. (2001). The attentive homunculus: Now you see it, now you don't. Neuroscience and Biobehavioral Reviews, 25, 477-496.

Pashler, H., Johnston, J. C., \& Ruthruff, E. (2001). Attention and performance. Annual Review of Psychology, 52, 629-651.

Periáñez, J. A., Maestú, F., Barcelo, F., Fernández, F., Amo, C., \& Ortiz Alonso, T. (2004). Spatiotemporal brain dynamics during preparatory set shifting: MEG evidence. Neuroimage, 21, 687-695.

Periáñez, J. A., Nyhus, E., \& Barcelo, F. (2005). Context updating and task-switching: An inquiry into the functional value of the novelty P3 potential. Psychophysiology, 42, S100.

Picton, T., Woods, D. L., Stuss, D. T., \& Campbell, K. (1978). Methodology and meaning of human evoked potential scalp distribution studies. In D. Otto (Ed.), Multidisciplinary perspectives in event-related brain potential research (pp. 515-522). Washington, DC: U.S. Goverment Printing Office.

Polich, J. (2003). Theoretical overview of P3a and P3b. Detection of change: Event-related potential and fMRI

findings (pp. 83-98). Boston: Kluwer Academic Publishing.

Posner, M. I. (Ed.) (2004). Cognitive neuroscience of attention. New York: The Guilford Press.

Ranganath, C., \& Rainier, G. (2003). Neural mechanisms 
for detecting and remembering novel events. Nature Reviews Neuroscience, 4, 193-202.

Ridderinkhof, K. R., van den Wildenberg, W. P., Segalowitz, S. J., \& Carter, C. S. (2004). Neurocognitive mechanisms of cognitive control: The role of prefrontal cortex in action selection, response inhibition, performance monitoring, and reward-based learning. Brain and Cognition, 56, 129-140.

Rubinstein, J. S., Meyer, D. E., \& Evans, J. E. (2001). Executive control of cognitive processes in task switching. Journal of Experimental Psychology: Human Perception and Performance, 27, 763-797.

Rushworth, M. F., Passingham, R. E., \& Nobre, A. C. (2002). Components of switching intentional set. Journal of Cognitive Neuroscience, 14, 1139-1150.

Rushworth, M. F., Walton, M. E., Kennerley, S. W., \& Bannerman, D. M. (2004). Action sets and decisions in the medial frontal cortex. Trends in Cognitive Sciences, 8, 410-417.

Ruthruff, E., Pashler, H. E., \& Klaassen, A. (2001). Processing bottlenecks in dual-task performance: Structural limitation or strategic postponement? Psychonomic Bulletin Reviews, 8, 73-80.

Sergent, C., Baillet, S., \& Dehaene, S. (2005). Timing of the brain events underlying access to consciousness during the attentional blink. Nature Neuroscience, 8 $1391-1400$.

Shallice, T. (2002). Fractionation of the supervisory system. In D. T. Stuss \& R. T. Knight (Eds.), Principles of frontal lobe function (pp. 261-277). New York: Oxford University Press.

Soltani, M., \& Knight, R. T. (2000). Neural origins of the P300. Critical Reviews in Neurobiology, 14, 199-224.

Squires, N. K., Squires, K. C., \& Hillyard, S. A. (1975). Two varieties of long-latency positive waves evoked by unpredictable auditory stimuli in man. Electroencephalography and Clinical Neurophysiology, 38, 387-401.

Stuss, D. T., \& Picton, T. W. (1978). Neurophysiological correlates of human concept formation. Behavioral Biology, 23, 135-162.

Sussman, E., Winkler, I., \& Schroger, E. (2003). Top-down control over involuntary attention switching in the auditory modality. Psychonomic Bulletin Reviews, 10, 630-637.

Yantis, S. (2000). Goal-directed and stimulus-driven determinants of attentional control [Tutorial]. In S. Monsell \& J. Driver (Eds.), Attention and performance XVIII: Control of cognitive operations (pp. 73-104). Cambridge: MIT Press. 